\title{
Cross-modal estimation of angular velocity
}

\author{
JAMES H. BROWH \\ U.S. ARMY MEDICAL RESEARCH LABORATORY
}

The exponent of 1.0 found previously for numerical magnitude estimates of angular velocity during passive rotation was validated by auditory cross-modality estimates. Acceleration intensities ranged from $3 \% \mathrm{sec}^{2}$ to $24 \% \mathrm{sec}^{2}$, with durations varying from $10 \mathrm{sec}$ to $80 \mathrm{sec}$. It was concluded that adaptation evident in responses to the longer duration stimuli is a real phenomenon and of potential significance as one form of disorientation.

Even though numerical magnitude estimation has been extensively used in a variety of scaling tasks (e.g., Stevens, 1957, 1958, 1966), it has only recently been applied to the assessment of subjective velocity during stimulation of the vestibular system by angular acceleration (Brown, 1966). Using a modification of the Stevens' scaling technique, Brown found an exponent on the order of 1.0 for the power function relating angular acceleration ( $\alpha$ t) and numerical subjective estimates of stimulus magnitude. This modification consisted of $S$ making frequent numerical responses during periods of angular acceleration and ensuing constant velocity in order to estimate the momentary magnitude of his rotary sensation. A finding of particular interest was that Ss evidenced considerable adaptation in their responses to accelerations of longer durations (48 and $80 \mathrm{sec})$. The extent of the adaptation contrasts markedly with both electrophysiological and nystagmic responses to similar stimulus configurations (Crampton, 1965; Jones, 1967). Neither nystagmic nor electrophysiological responses appear to be as susceptible to adaptation as does the subjective response. However, in view of the extremely long duration of these accelerations and the frequent, repetitive numerical responses, response stereotypy could have influenced S's numerical estimations. That is, S's responses could have reflected a tendency to give the next higher or lower number not as a function of an increase or decrease in the actual sensation but rather as a function of the rate of increase or decrease in previous responses. If in fact stereotypy significantly influenced S's responses, much of the adaptation evident in the subjective responses to the longer duration accelerations would have been artifactual. Erroneously reporting a decrease in angular velocity when in fact velocity is increasing at a low but constant rate (adaptation) is a general type of disorientation that might influence a pilot's judgment. The nature and extent of such adaptation should be precisely specified.

In order to mitigate the potential influence of re- sponse stereotypy, as well as to validate the exponent of 1.0 found previously for numerical estimates of subjective velocity (Brown, 1966), the present study required $S$ to make a cross-modal estimate of angular velocity by continuously matching the loudness of an auditory signal to his sensed angular velocity.

\section{Apparatus \\ METHOD}

The hydraulically driven angular accelerator, described in detail by Brown and Crampton (1964), consists of a rotating crossbeam supporting a chair. A closed, light-proof canopy eliminated cues to angular velocity associated with air movement across the face, and permitted control of visual stimulation. An adjustable bite board restrained $S$ 's head in a fixed position over the axis of rotation. The ventilating fan for the capsule interior provided masking noise at a low, but sufficient level to prevent detection of extraneous signals coupled to actual machinery rotation. Auditory signals were provided by a white noise generator and passed through instrument slip rings to a set of binaural earphones. $S$ varled the intensity of the signal during each trial using a single-turn potentiometric control. A $6 \mathrm{~V}$ dc signal was also imposed on the control unit such that the voltage varied systematically from 0 to $6 \mathrm{~V}$ as $\mathrm{S}$ increased the intensity of the auditory signal. The 0 to $6 \mathrm{~V}$ signal was led out through slip rings, converted to a digital output, and printed on paper tape to provide a second-by-second monitoring of the intensity adjustments made by $S$. Electronic voice communication between $S$ and $E$ was provided.

\section{Subjects}

Twenty male Ss, ranging from 18 to 22 years, were selected from a population of $30 \mathrm{Ss}$ who had participated in previous experiments involving repeated exposure to constant angular acceleration. All Ss were in excellent health, and none had any previous history of auditory or labyrinthine difficulty.

\section{Procedure}

Each $S$ received nine trials on each of eight experimental sessions. An initial practice session consisted of 10 trials to acquaint $S$ with procedural details and to familiarize him with the range of accelerative stimuli. Each trial consisted of a period of angular acceleration followed by constant velocity. A 5 min intertrial interval of constant velocity was programmed to avoid interactive effects from suc- 

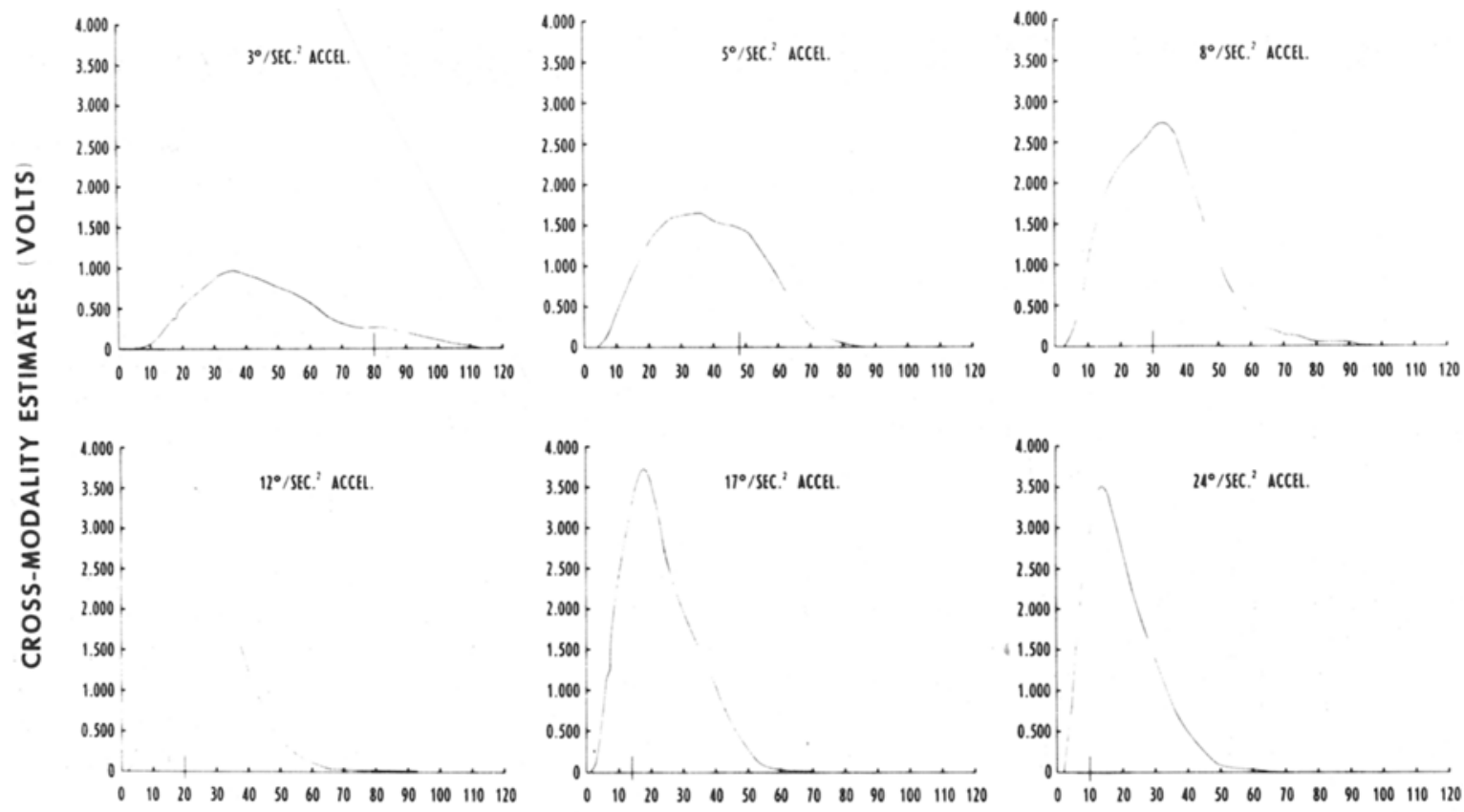

TIME SEC.

Fig. 1. Geometric mean cross-modal estimates (volts) plotted over sec for each of the six angular accelerations. All accelerations were initiated at 0 sec and maintained until termination of the acceleration as indicated by the vertical bar on the baseline.

cessive accelerations. All trials were conducted in total darkness. Each acceleration was presented symmetrically around zero velocity to minimize the influence of both the slight gravitational force exerted on those parts of the body not directly in the axis of rotation and the bearing and machinery noises that became evident at higher RPM. For example, at the start of the session, $S$ received a low-level acceleration to a constant velocity of $20 \mathrm{RPM}$ in a CCW direction. With the onset of the first trial, $S$ was accelerated through zero velocity to a terminal velocity of $20 \mathrm{RPM}$ in a $\mathrm{CW}$ direction. After a period of $5 \mathrm{~min}$, the next acceleration, negative in sign, returned $S$ to the initial CCW velocity of $20 \mathrm{RPM}$. The test trials consisted of angular accelerations $(\alpha)$ of $3.0^{\circ}, 5.0^{\circ}, 8.0^{\circ}, 12.0^{\circ}, 17.0^{\circ}$, and $24.0^{\circ} / \mathrm{sec}^{2}$ maintained for durations ( $t$ ) from 10 to $80 \mathrm{sec}$ such that the $t$ products involved a total velocity change of $40 \mathrm{RPM}$ (20 RPM through zero velocity to $20 \mathrm{RPM}$ ). A standard acceleration of $90 / \mathrm{sec}^{2}$ and $10 \mathrm{sec}$ duration was introduced on every third trial. During the standard trial, $S$ was instructed not to respond. Precisely at the end of this $10-\mathrm{sec}$ acceleration, $E$ presented a $1-\mathrm{sec}$ auditory reference stimulus (80 dB SPL). During test trials, $S$ used the potentiometer to adjust the intensity $(60-110 \mathrm{~dB})$ of the white noise signal to match the growth and decline of his subjective angular velocity. The same random ordering of test trials within blocks of the six test stimuli was followed for all Ss. Thus, each S received eight repetitions of each of the six test stimuli.

\section{RESULTS AND DISCUSSION}

Second-by-second logarithmic transformations of the voltage readouts were made for the responses of each $S$ to every test stimulus. Geometric means for each second of the six text stimuli were computed across replications and Ss ( 8 replications by $20 \mathrm{Ss}$ $=160$ values contributing to each mean). The secondby-second geometric means for each of the six test stimuli are plotted against time in Fig. 1. All accelerations were initiated at $0 \mathrm{sec}$ and terminated at the time indicated by the vertical bar on the baseline. Considerable adaptation, i.e., a decrease in subjective velocity before stimulus termination, is clearly evident for the least intense but longest duration accelerations $\left(3^{\circ}\right.$ and $\left.5^{\circ} / \mathrm{sec}^{2}\right)$. All Ss showed comparable and considerable adaptation to these low intensity accelerations for all test sessions. Also, as found previously, the subjective estimates peak with the stimulus for the middle intensities $\left(8^{\circ}\right.$ and $12^{\circ} / \mathrm{sec}^{2}$ ) while there is considerable overshoot in responses to the more intense accelerations $\left(17^{\circ}\right.$ and $24^{\circ} / \mathrm{sec}^{2}$ ). These similarities between the numerical and cross-modal magnitude estimates suggest that response stereotypy is not a significant factor. Adaptation to long-duration accelerative stimulation is a very real phenomenon and potentially hazardous as one form of disorientation. Although vestibularly induced disorientation is generally viewed only as a product of very intense accelerations, it is apparent that disorientation can result from even very weak stimulation, if it is maintained for long periods. 
This adaptation presents problems for the biophysical model of the semicircular canals, a torsion pendulum analogue (van Egmond, Groen, \& Jongkees, 1949). If adaptation is assumed to be a peripheral process, modification of the constants for elasticity, friction and/or mass is clearly required.

The log-log plot in Fig. 2 clearly shows that the equation and exponent of 1.0 derived previously from the numerical magnitude estimation data provide an excellent fit for the present data. As in the previous study, only data in which adaptation was not a confounding factor are plotted.

Both nystagmic and subjective responses to angular acceleration show systematic reductions in their intensity and duration with repeated exposure (e.g., Crampton, 1964; Dodge, 1923; Griffith, 1920). For example, athletes, skaters, pilots, and others in special occupations involving repeated exposure to acceleration environments have habituated responses (Aschan, 1954; Mowrer, 1934), In order to control for habituation, that is, to prevent decreases in sensitivity during the course of the present study, all Ss were given repeated exposures to angular accelerating prior to initiating the scaling sessions The most intense acceleration in the present study, $24^{\circ} / \mathrm{sec}^{2}$, is the stimulus to which Ss would be most likely to show response decrements if habituation were not adequately controlled. In Fig. 3 geometric means computed across Ss are plotted for the eight repetitions of this stimulus. In view of no systematic decrement in responses across trials, it can be concluded that these Ss were successfully prehabituated and that habituation is not an interacting factor in the present experiment. This raises the intriguing question of whether the responses of experientially naive Ss could also be described by the

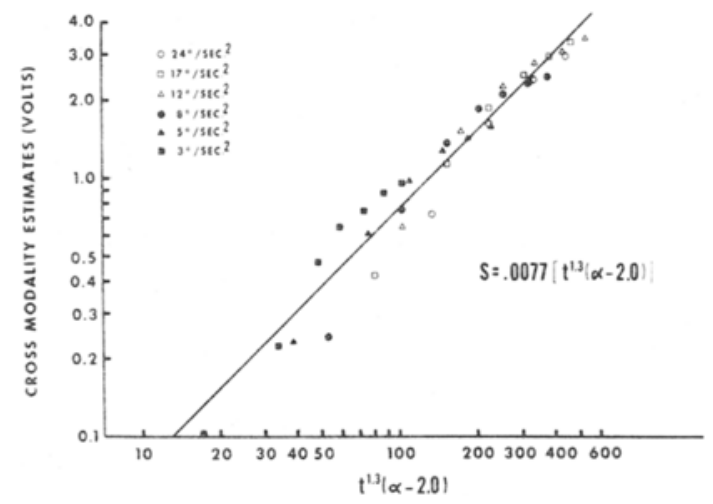

Fig. 2. Geometric mean cross-modal estimates plotted logarithmically against the logarithmic $t$ a guantity (time, $t$, represents time in sec from the onset of the stimulus while acceleration, at, is expressed in degrees $/ \sec ^{2}$ ).

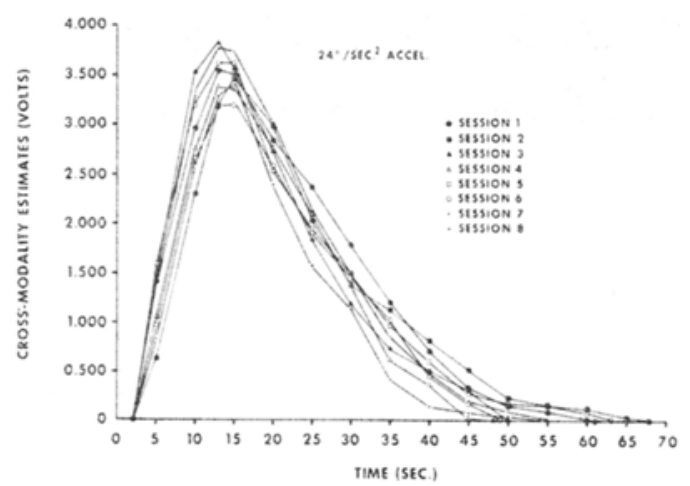

Fig. 3. Geometric mean cross-modality estimates plotted over sec for the eight successive repetitions of the $24 \% \mathrm{sec}^{2}$ acceleration.

exponent of 1.0 which is clearly derived from a habituated population. In view of Aschan's comparative work with both aviators and normal Ss (Aschan, 1954), it appears that different exponents will be required for different levels of habituation. If this assumption is verified, it is concelvable that the exponent itself would serve as a useful and meaningful index to describe the level of habituation of an individual.

\section{References}

Aschan, G. Response to rotatory stimuli in fighter pilots. Acta Otolaryng. (Stockholm), 1954, Suppl. 116.

Brown, J. H. Magnitude estimation of angular velocity during passive rotation. J. exp. Psychol., 1966, 72, 169-172.

Brown, J. H., \& Crampton, G. H. Quantification of the human nystagmic response to angular acceleration. Prediction formulae and nomograph. Acta Otolaryng. (Stockholm), 1964, 58, 555-564.

Crampton, G. H. Response of single cells in cat brainstem to angular acceleration in the horizontal plane. NASA SP-77, 1965, 85-96.

Crampton, G. H. Habituation of ocular nystagmus of vestibular origin. In M. B. Bender (Ed.), The Oculomotor System. New York: Hoeber, 1964

Dodge, R. Habituation to rotation. J. exp. Psychol., 1923, 6, 1-35.

Griffith, C. R. The organic effects of repeated bodily rotation. J. exp. Psychol, 1920, 3, 15-46.

Jones, $\mathbf{G}$. $\boldsymbol{M}$. On the dependence of oculo-motor response upon the neural response of the vestibular system. In Third symposium on the role of the vestibular organs in space exploration. Vol. 3 , Pensacola, Florida, NASA, 1967.

Mowrer, $\mathbf{O}$. H. The modification of vestibular nystagmus by means of repeated elicitation. Comp. Psychol. Monogr., 1934, 9, 1-48.

Stevens, S. S. On the operation known as judgment. Amer. Scient., $1966,54,385-401$.

Stevens, S. S. Problems and methods of psychophysics. Psychol. Bull., 1958, 55, 177-196.

Stevens, S. S. On the psychophysical law. Psychol. Rex., 1957 , $64,153-181$.

van Egmond, A. A. I., Groen, J. J., \& Jongkees, L. B. W. The mechanics of the semicircular canal. J. Physiol. (London), 1949, $110,1-17$

(Accepted for publication October 22, 1967.) 